\title{
Bacterial Hash Function Using DNA-Based XOR Logic Reveals Unexpected Behavior of the LuxR Promoter
}

\author{
Brianna Pearson ${ }^{1, \pm}$, Kin H. Lau ${ }^{1, \neq}$, Alicia Allen ${ }^{2}$, James Barron ${ }^{1,3}$, Robert Cool ${ }^{2}$, Kelly Davis ${ }^{4}$, Will DeLoache ${ }^{1}$, \\ Erin Feeney ${ }^{1}$, Andrew Gordon ${ }^{2}$, John Igo $^{5}$, Aaron Lewis ${ }^{5}$, Kristi Muscalino ${ }^{4}$, Madeline Parra ${ }^{4}$, Pallavi Penu- \\ metcha $^{1}$, Victoria G. Rinker ${ }^{1,6}$, Karlesha Roland ${ }^{1,7}$, Xiao Zhu' ${ }^{2}$, Jeffrey L. Poet ${ }^{5,8}$, Todd T. Eckdahl ${ }^{2,8}$, Laurie J. \\ Heyer $^{4,8}$ and A. Malcolm Campbell ${ }^{1,8,}$
}

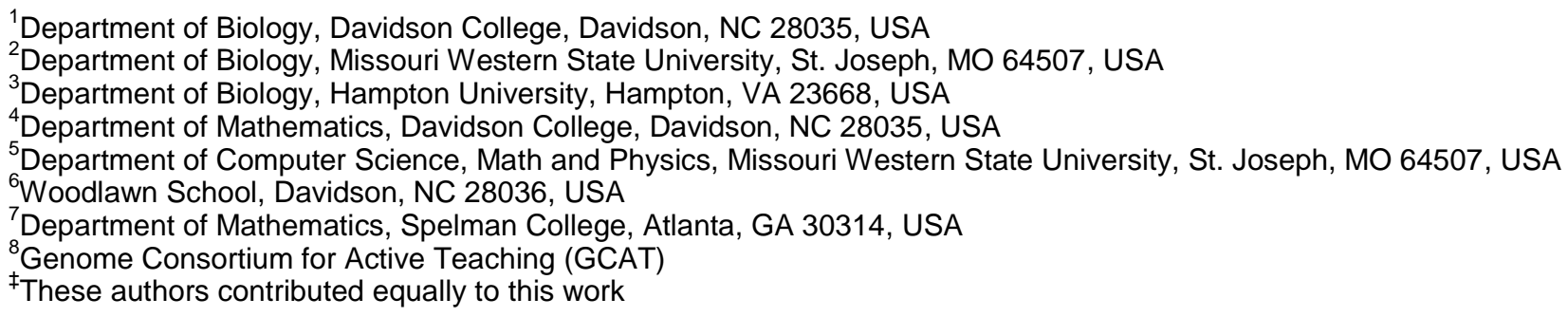

Subject areas: Synthetic biology, Biological computation/Database, Biomathematics/Mathematical Biology and Medicine

Author contribution: Poet, Eckdahl, Heyer and Campbell conceived of the project and supervised the student' project and supervised the students work; DeLoache did the time-delayed growth experiments and movie; Lau, Allen, Barron, Cool, Davis, DeLoache Feeney, Gordon, Lewis, Penumetcha, Rinker and Zhu performed the wet lab experiments and analyzed the data; Davis, Igo, Muscalino, Parra and Roand designed the hash function and did all the mathematical work; Pearson, Eckdahl and Campbell wrote the manuscript, with Poet and Heyer editing and improving the writing.

${ }^{*}$ Correspondence and requests for materials should be addressed to A.M.C. (macampbell@davidson.edu).

Reviewer: J. Christopher Anderson UC Berkeley, USA; Sven Panke, ETH

Editor: Keun Woo Lee, Gyeongsang National University, Republic of Korea

Received July 08, 2011

Accepted July 15, 2011;

Published July 18, 2011

Citation: Pearson, B., et al. Bacterial Hash Function Using DNA-Based XOR Logic Reveals Unexpected Behavior of the LuxR Promoter. IBC 2011, 3:10, 1-8.

doi: 10.4051 /ibc. 2011.3.3.0010

Funding: Support is gratefully acknowledged from NSF UBM grant DMS 0733952 to Davidson College and DMS 0733955 to Missouri Western State University, HHMI grants 52005120 and 52006292 to Davidson College, the James G. Martin Genomics Program at Davidson College, and the Missouri Western State University Foundation and Summer Research Institute.

Competing interest: All authors declare no financial or personal conflict that could incappropriately bias their experiments or writing. their experiments or writing.

Copyright: This article is licensed under a Creative Commons Attribution License, which freely allows to download, reuse, reprint, modify, distribute, and/or copy articles as long as a proper citation is given to the original authors and sources.

\section{SYNOPSIS}

Introduction: Hash functions are computer algorithms that protect information and secure transactions. In response to the NIST's "International Call for Hash Function", we developed a biological hash function using the computing capabilities of bacteria. We designed a DNA-based XOR logic gate that allows bacterial colonies arranged in a series on an agar plate to perform hash function calculations.

Results and Discussion: In order to provide each colony with adequate time to process inputs and perform XOR logic, we designed and successfully demonstrated a system for time-delayed bacterial growth. Our system is based on the diffusion of B-lactamase, resulting in destruction of ampicillin. Our DNA-based XOR logic gate design is based on the opposition of two promoters. Our results showed that $P_{\text {lux }}$ and $P_{O m p C}$ functioned as expected individually, but $\mathrm{P}_{\text {lux }}$ did not behave as expected in the XOR construct. Our data showed that, contrary to literature reports, the $\mathrm{P}_{\text {lux }}$ promoter is bidirectional. In the absence of the 30 66 inducer, the LuxR activator can bind to the $\mathrm{Plux}_{\text {lux }}$ promoter and induce backwards transcription.

Conclusion and Prospects: Our system of time delayed bacterial growth allows for the successive processing of a bacterial hash function, and is expected to have utility in other synthetic biology applications. While testing our DNA-based XOR logic gate, we uncovered a novel function of $P_{\text {lux. }}$ In the absence of autoinducer $30 \mathrm{C} 6$, LuxR binds to $P_{\text {lux }}$ and activates backwards transcription. This result advances basic research and has important implications for the widespread use of the $\mathrm{P}_{\text {lux }}$ promoter.

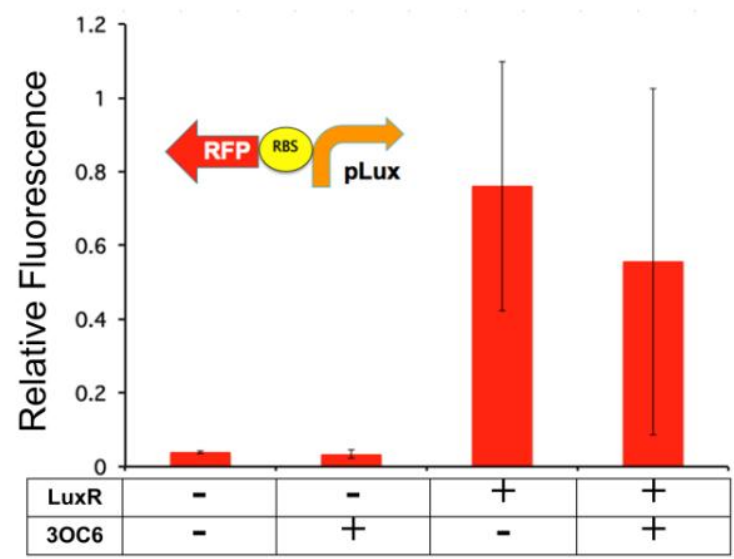

Keywords: hash function, time-delayed bacterial growth, DNA-based XOR logic gate, Plux, LuxR, POmpC, bidirectional promoter, synthetic biology 


\section{Introduction}

Protection of electronic communication is vital to the economic and defense capabilities of our nation. "America's Next Top Hash Function" competition, as Wired Magazine describes it, recognized the need for a novel hash function in light of recent attacks on the integrity of the current standard and challenged the global community to design a secure hash function standard ${ }^{1}$. In late 2012, the U.S. National Institute of Standards and Technology (NIST) will conclude their international competition for the development of a new and improved hash function and a winner will be chosen from five finalists. The competition was introduced in November 2007 in response to concerns that the current standard, SHA-1 (Secure Hash Algorithm) was out-of-date and not secure. A secure cryptographic hash algorithm is essential to authenticate electronic documents and maintain their integrity. Hash functions also protect passwords, software, and monetary transactions from hackers ${ }^{2}$. A hash function encrypts an input of arbitrary length into a "message digest" code of fixed small size in a way that is irreversible, meaning the input cannot be deduced from the output. The hash output value of a given input string is its "signature" or "fingerprint" that works to detect document tampering ${ }^{3}$. The ideal hash function must satisfy three properties: preimage resistance, second preimage resistance, and collision resistance. Preimage resistance refers to the difficulty of finding an input that hashes to a pre-specified output. Second preimage resistance describes the difficulty of identifying a second input that hashes the same output as a given input. Collision resistance is a measure of the likelihood that the same hash value is produced by two distinct inputs. A good hash function is completely irreversible and designed so that collisions are as rare as possible. In addition, it should be quick and easy to extract an output from a given input message ${ }^{4}$.

In response to the call for a reliable and novel hash function, we developed a biological hash function to be implemented inside living bacteria. Bacterial computers provide a unique alternative technology to silicon computers. Cellular computers have the advantage of exhibiting enormous parallel computing capabilities, intercellular communication, ability to interface with the biological world, and reusability ${ }^{5,6}$. Computing efficiency may result from the use of single analog logic gates in a population of bacterial computers, as opposed to the thousands of gates required in digital processes used by conventional computers ${ }^{7}$. Furthermore, computer hackers are untrained in decoding biological computers. We chose the XOR (exclusive OR) logic gate to execute our biological hash function. Among the basic Boolean logic operators, only the XOR and NXOR gates possess an equal chance for an output of 0 or $1^{8}$. In an XOR logic gate, an output is produced if and only if exactly one of the binary inputs is present. If both inputs are present, or both inputs are absent, the logic gate does not produce an output.

As a proof-of concept, we designed a simple linear hash function out of a sequential series of bacterial colonies, each of which performs XOR logic on inputs consisting of one bit from the message and one bit from the output of the previous colony. In addition to this simple linear model, we designed several more complex, three dimensional, and detailed alternative hash functions ${ }^{9}$. As shown in Figure 1, the first colony uses XOR logic to respond to two chemical inputs, one in the form of a key and the other as the first part of the input message. The output from the first colony is used as input for the second colony, along with the next bit of the input message. These two inputs are processed by XOR logic in the second colony and the output is passed on to each successive colony in the series. The final colony output determines the hash value of the input "message".

Encoding an XOR logic gate in a biological system necessitates that a cell differentially respond to an input depending on whether

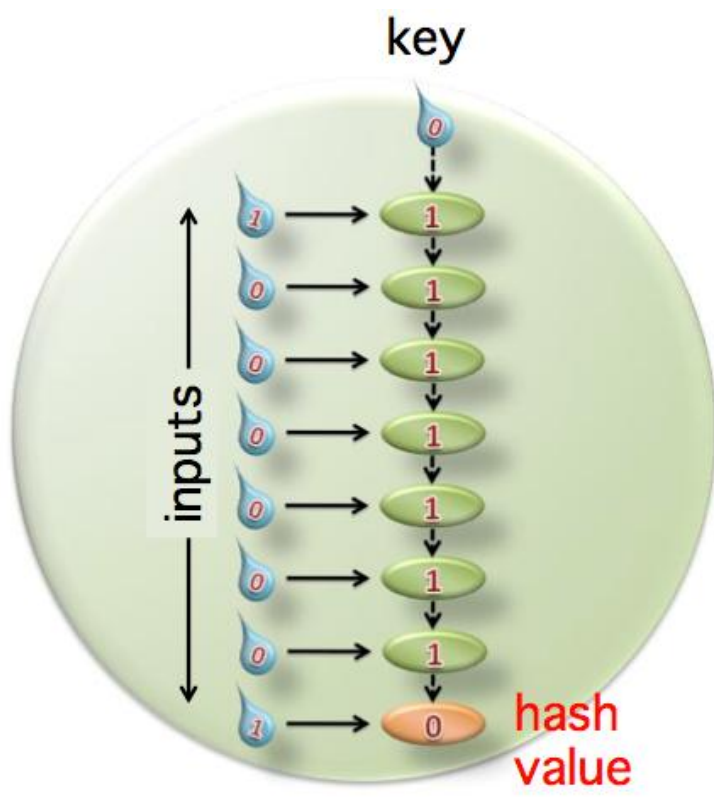

Figure 1. Proof-of-concept bacterial hash function. Colonies (green) grow in a time-delayed manner. The key (blue drop on top) and the binary input message (blue drops on left) are applied manually. Each colony receives two bits of input. The colonies respond sequentially with XOR logic to inputs and determine whether or not to generate an input to the next colony in the chain. The final colony in the chain will determine the hash value of the input message by expression of a fluorescent reporter.

the input is presented alone or in combination with another input. Differential response to an input presents a formidable challenge, which explains why engineering of a direct DNA-based XOR gate has not been reported. The assembly of a chemistry-based XOR logic gate has been reported, with amines and protons as inputs ${ }^{10}$ Voigt, et al. (2011) recently assembled an indirect DNA-based XOR gate but only through a combinatorial circuit consisting of three NOR gates, a buffer gate, and a specific spatial arrangement on the agar plate ${ }^{11}$. Stojanovic, et al. (2002) designed an in vitro "deoxyribozyme-based" XOR gate consisting of single-stranded oligonucleotides of switched loops acting as inputs and cleaved oligonucleotide products as the output ${ }^{12}$. In contrast to XOR gates, researchers have been able to successfully construct and implement other DNA-based Boolean logic gates in vivo, including AND, OR, NOT, NOR and NAND logic ${ }^{13}$.

Our goal was to design and test a direct DNA-based XOR logic gate that could be used in a series of bacterial colonies to implement a hash function. XOR logic requires that the presence of two inputs produces no output (Figure 2). In order to accomplish this result in a biological system, we selected two inducible promoters to be placed in opposition to one another. When both promoters are induced, transcription would be blocked by the binding of their respective DNA-binding proteins and RNA polymerases. One of the promoters is the $P_{\text {lux }}$ promoter, a well-documented and widely used synthetic biology part from the LuxR quorum sensing mechanism. One of the first quorum sensing systems discovered, the luxR operon was isolated from $V$. fischeri, a marine bacterium living symbiotically within the squid Euprymna scolopes ${ }^{14}$. According to the literature ${ }^{14,15}$ the transcriptional activator protein LuxR must first bind to its ligand, the chemical autoinducer $3-0 \times 0-\mathrm{C}_{6}$-homoserine lactone (30C6), before LuxR can bind to, and subsequently activate, the promoter $\mathrm{P}_{\text {lux. }}$ The autoinducer $30 \mathrm{OC} 6$ is synthesized by the bacterially encoded Luxl enzyme. Once $\mathrm{P}_{\text {lux }}$ is activated, the luxl gene is transcribed at a high rate, initiating a positive feedback autoinduction circuit. For the opposing promoter, we utilized the ompC/envZ signaling system ${ }^{16}$. $P_{\mathrm{OmpC}}$ is part of the endogenous osmotic stress 


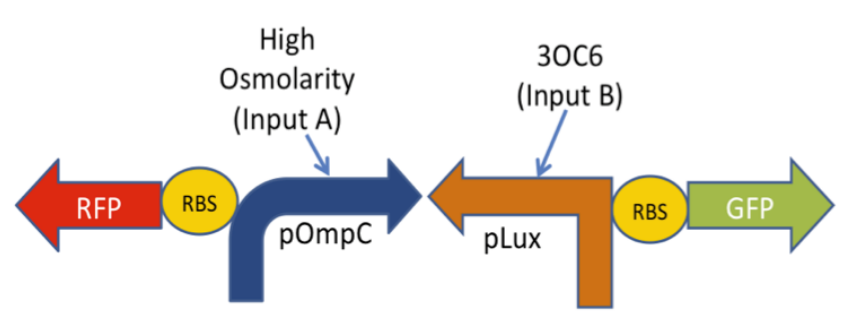

\begin{tabular}{|c|c|c|}
\hline $\begin{array}{c}\text { High Osmolarity } \\
\text { (Input A) }\end{array}$ & $\begin{array}{c}30 \mathrm{CC} \\
\text { (Input B) }\end{array}$ & $\begin{array}{c}\text { Fluorescence } \\
\text { (Output) }\end{array}$ \\
\hline 0 & 0 & 0 \\
\hline 1 & 0 & 1 (GFP) \\
\hline 0 & 1 & 1 (RFP) \\
\hline 1 & 1 & 0 \\
\hline
\end{tabular}

Figure 2. DNA-based XOR logic gate design and XOR truth table. A) The two inputs are high osmolarity and the $30 C 6$ autoinducer. Predicted XOR outputs are red fluorescence with the $30 \mathrm{OC} 6$ input, green fluorescence with the high osmolarity input, or no fluorescence with both inputs or neither input. B) Truth table summarizes XOR logic gate.

response unit in $E$. coli that becomes activated in media of high osmolarity. Wild-type $E$. coli cells possess the outer membrane pore proteins, $\mathrm{OmpF}$ and $\mathrm{OmpC}$, that are reciprocally regulated by the osmolarity of their surrounding environment. In high osmolarity media made with the addition of $\mathrm{NaCl}$, such as $\mathrm{LB}$, ompC is transcribed and cells accumulate the smaller OmpC passive diffusion pores in their membranes. In low osmolarity media, such as TY, $o m p F$ encodes a large diameter pore. A histidine kinase protein, EnvZ, monitors osmolarity in the vicinity of the cell and phosphorylates a transcription factor, OmpR, and phospho-OmpR promotes transcription of ompC via its $\mathrm{P}_{\mathrm{OmpC}}$ promotor $^{17-19}$. In the context of the XOR gate, the addition of 3OC6 to the media serves as one input while the addition of $\mathrm{NaCl}$ (high osmolarity LB media) serves as the other input. When both inputs are present or when both are absent, the logic gate is predicted to return no output. The XOR gate will be encoded into each of the colonies depicted in Figure 1. The hash function key will take the form of the presence or absence of $30 \mathrm{OC} 6$ and will be one of the two inputs to the XOR function of the first colony. The other input will be the presence or absence of $\mathrm{NaCl}$ administered adjacent to the first colony, representing the first part of the message. The first colony will process these two inputs and deliver an output in the form of the presence or absence of 30C6 to the second colony. The second XOR input for this colony will be the presence or absence of $\mathrm{NaCl}$ administered adjacent to the second colony, representing the second part of the message. In this way, successive colonies will process the message, with the output from the previous colony as the presence or absence of $30 \mathrm{OC} 6$ connected to the expression of a reporter gene such as RFP in the final colony.

The design of our DNA-based XOR gate was based on an understanding of the molecular mechanisms by which the two promoters function, as documented in the literature. The two promoters functioned as expected when tested individually, but we detected aberrant behavior when testing the complete XOR configuration with opposing promoters. Frequently in synthetic biology, devices function as designed and the project is deemed successful. However, synthetic biology devices occasionally fail due to incomplete understanding about parts that are central to the design. It is important that synthetic biologists learn to investigate the reasons for failed designs in the interest of basic research and the purpose of redesign. In this case, we discovered that the transcription factor LuxR and the $P_{\text {lux }}$ promoter do not function as previously reported ${ }^{14,15}$. This result contributes to basic research that complements our successful demonstration of time-delayed bacterial growth, which is a contribution to applied research.

\section{Results and Discussion}

In order for the XOR-based hash function to process information over time, each colony in the series must perform its logic sequentially. If all colonies grew simultaneously, the hash function would fail because colonies at the end of the chain would perform their XOR logic before the input from the previous colony had reached them. Thus, we needed to devise a mechanism of time-delayed growth so that colonies would sequentially process their inputs and pass along their output to the next colony before the following colony had grown and performed XOR logic. A literature search failed to uncover a technique to accomplish time-delayed growth in a simple and inexpensive way. We took advantage of the often unintended consequence of satellite colonies forming when transformed colonies are left on an ampicillin selection plate too long ${ }^{20}$. Following a bacterial cell transformation, satellite colonies can form around resistant colonies containing cells that successfully integrated an ampicillin-resistance plasmid. Ampicillin is often mistakenly thought to kill E. coli outright but ampicillin and other B-lactams prevent cell wall peptidoglycan synthesis by the competitive inhibition of a transpeptidase $e^{21}$, thereby preventing the bacteria from forming new cell walls during cell division. Ampicillin-resistant cells can secrete up to $90 \%$ of the enzyme B-lactamase beyond the periplasmic space when the enzyme is highly expressed ${ }^{22}$. The enzyme cleaves the B-lactam ring of ampicillin and inactivates the antibiotic. Nonampicillin-resistant satellite colonies grow because ampicillin is deactivated surrounding the B-lactamase secreting cells and viable ampicillin-sensitive cells can thrive once again after hours of no growth. We exploited this often undesirable artifact to produce timedelayed colony growth. Previous research suggested that Blactamase diffuses slowly through LB agar, thus providing a means to control the growth of colonies in a time-dependent manner ${ }^{23}$. We successfully demonstrated time-delayed growth produced by Blactamase diffusion (Figure 3). An inoculant of ampicillin-resistant bacteria was placed at one edge of an agar plate with ampicillinsensitive bacteria inoculated every $0.5 \mathrm{~cm}$ along a line in each of three directions. As the B-lactamase secreted by the ampicillinresistant bacteria diffused across the plate, ampicillin-sensitive bacteria at increasing distances from the ampicillin-resistant bacteria were able to grow. The spread of colonies over time indicates
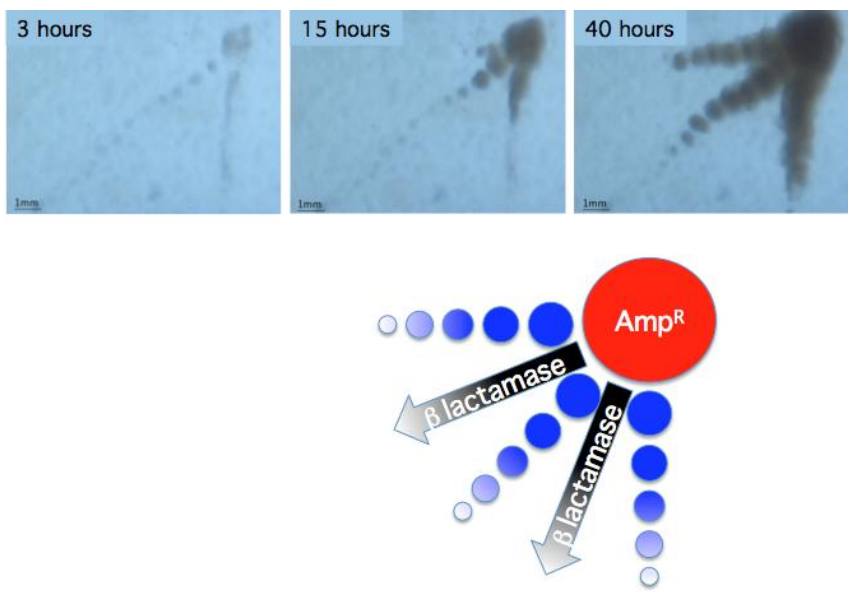

Figure 3. Time-delayed bacterial growth. A) Screen shots from timedelayed colony growth at 3 hours, 15 hours, and 40 hours on an LB agar plate with $100 \mu \mathrm{g} / \mathrm{mL}$ ampicillin of. (Complete movie is available online.) B) Schematic drawing of time-delayed growth experiment. An ampicillin-resistant colony was inoculated in the top right corner of the image with ampicillin-sensitive inoculants placed in three linear paths away from the ampicillin-resistant colony. 
that B-lactamase was conferring ampicillin-resistance to colonies sequentially and uni-directionally. (A video of this time-delay growth can be accessed online)

Time-delayed growth could allow for temporal tuning of a bacterial hash function since successive colonies would be given adequate time to process their XOR logic. We characterized our timedelayed colony growth system further and investigated what variables could be manipulated to govern the rate of colony growth. We measured the rate of ampicillin inactivation as a function of three variables: initial ampicillin concentration, agar concentration, and temperature (Figure 4). Over the course of three days, the appearance of colonies was linear. Ampicillin-sensitive colonies appeared faster with lower concentrations of ampicillin, as expected (Figure 4A). We hypothesized that higher agar concentrations would slow the diffusion of B-lactamase across the plate and therefore slow the ability of colonies to grow. Contrary to our expectations, higher agar concentration produced faster colony appearances (Figure 4B). Temperature also had an interesting effect on the appearance of non-resistant colonies. At a high concentration of ampicillin $(100 \mu \mathrm{g} / \mathrm{mL})$, there was no significant difference in the rate of delayed growth between the $30^{\circ} \mathrm{C}$ and $37^{\circ} \mathrm{C}$ (compare Figures $4 \mathrm{~A}$ and $4 \mathrm{C}$ ). However, at a lower ampicillin concentration (25 $\mu \mathrm{g} / \mathrm{mL}$ ), appearance of colonies was 1.5 times faster at $37^{\circ} \mathrm{C}$ (compare Figures $4 \mathrm{~A}$ and $4 \mathrm{C}$ ).

To test the function of our proposed XOR-based hash function, we built the construct depicted in Figure 2A. By arranging the promoters of the OmpR and LuxR signaling system head-to-head and coupling each to a distinct fluorescent protein marker, we could determine if XOR logic was processed inside live E. coli cells (Figure $2 \mathrm{~B}$ ). When cells are grown in high salt LB media and without the addition of 30C6, $P_{O m p C}$ should be activated and cells should fluoresce green. When $30 C 6$ is added to a low salt TY media, $P_{\text {lux }}$ should be activated and cells producing LuxR should fluoresce red. When cells are grown in low salt TY media in the absence of $30 \mathrm{C6}$, or in high salt LB media in the presence of $30 \mathrm{C} 6$, cells should not fluoresce at all.

Figure $5 \mathrm{~A}$ shows the results of experiments to test the function of the XOR gate. We measured RFP and GFP fluorescence under all four combinations of inputs in the presence of the LuxR regulatory protein. In the absence of LuxR, we expected that the $\mathrm{P}_{\text {lux }}$ promoter would not function (Figure 5B). No expression of the RFP gene is expected, and none was observed. We predicted that the absence of LuxR would have no effect on the ability of $P_{\text {ompc }}$ to respond to the high osmolarity input of $\mathrm{NaCl}$ in the $\mathrm{LB}$ media and that is what we observed. The observation of GFP expression when both the $30 \mathrm{O} 6$ and high osmolarity inputs were provided was consistent with the explanation that LuxR was not available to activate transcription form the $\mathrm{P}_{\text {lux }}$ promoter. In the presence of the LuxR regulatory protein and absence of both inputs, we observed unexpected GFP expression. In the presence of LuxR and the $30 \mathrm{OC} 6$ input combined with the absence of the high osmolarity $\mathrm{NaCl}$ input, we observed RFP expression as predicted. The result of GFP expression in the presence of the high osmolarity $\mathrm{NaCl}$ input and the absence of the 3066 input was also in accord with our expectations. When both inputs were present, we observed lower levels of unpredicted GFP expression. The presence or absence of both inputs proved to be the conditions that produced the unexpected behaviors when LuxR was expressed inside the cells.

In order to explain the unexpected behaviors of GFP expression, we hypothesized that the $P_{\text {lux }}$ promoter was stimulating backward transcription in the presence of LuxR but the absence of the $30 \mathrm{C} 6$ autoinducer. The results of experiments to test this hypothesis are show in Figure $5 \mathrm{C}$. The test construct was designed to result in RFP expression only when backwards transcription is supported by the BioBrick part containing the $\mathrm{P}_{\text {lux }}$ promoter. In the absence of LuxR, the addition of $30 \mathrm{OC} 6$ did not result in backwards transcription. However, the same construct, with the addition of the LuxR protein, had a very similar level of fluorescence with or without the addition of 30C6. Although there is less transcription of RFP in the presence of $30 \mathrm{O} 6$ than in the absence of $30 \mathrm{C6}$, the differences are not significant. Perhaps the lower average and larger error bars is due to stochastic binding of $30 \mathrm{C} 6$ and the resulting inconsistent forward vs. backward transcription from the same promoter. The literature indicates that LuxR binds $P_{\text {lux }}$ only after $30 C 6$ binds to $\operatorname{LuxR}^{14,15,24-26}$. Our results show that the BioBrick part containing $P_{\text {lux }}$ supports backwards transcription when LuxR is present in the cell and 30C6 is absent. The backwards transcription from $\mathrm{P}_{\text {lux }}$ in the presence of LuxR also explains the failure of the XOR gate to perform its logical operations. It is worth noting that the $\mathrm{P}_{\text {lux }}$ promoter used in this study contains only the -10 and -35 regions with a lux box but not the additional $70 \mathrm{bp}$ portion of DNA found in $V$. fischeri that normally leads to the transcription of $\operatorname{LuxR}^{24}$.

\section{Conclusion and Prospects}

Our design of a biological hash function using a direct DNAbased XOR logic gate was a novel response to the international call for a new and improved standard hash function ${ }^{1,2}$. Our bacterial hash function provided a new approach for a robust and secure
(A)

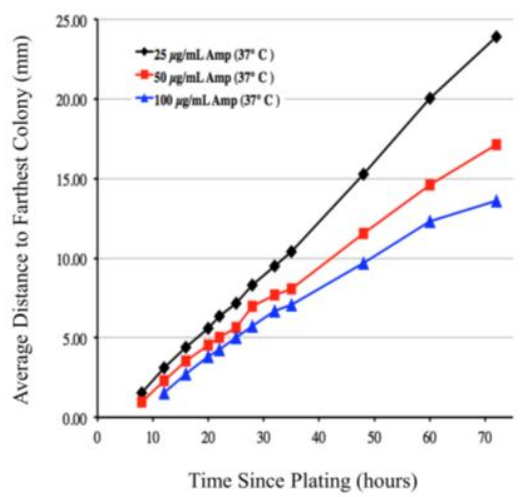

(B)

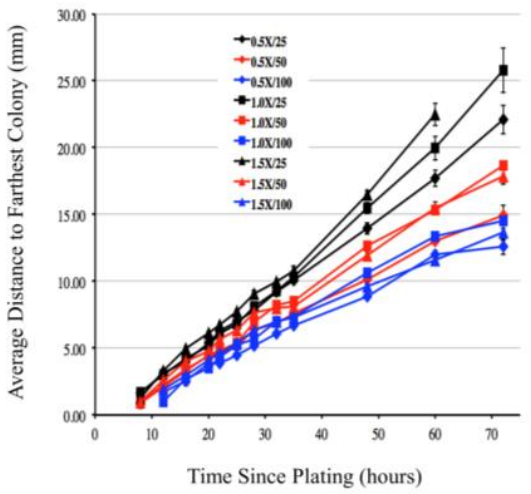

(C)

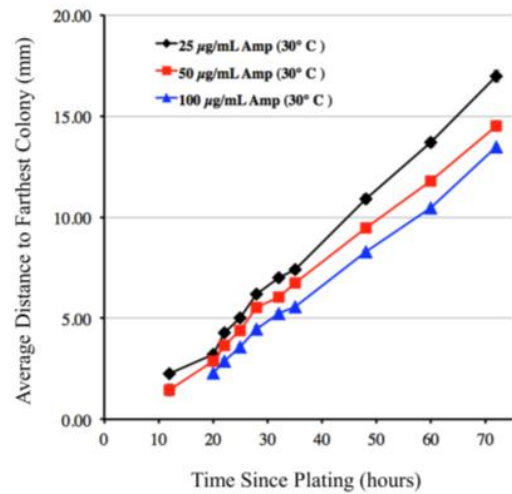

Figure 4. Bacterial growth rate. A) The effect of ampicillin concentration on growth rate in time-delayed growth at $37^{\circ} \mathrm{C}$. B) Effect of agar concentration on bacterial growth rate at $37^{\circ} \mathrm{C}$. Media are described by agar concentration $(0.5 \mathrm{x}=7.75 \mathrm{~g} / \mathrm{L})$ over ampicillin concentration $(25=25 \mu \mathrm{g} / \mathrm{mL})$. C) The effect of ampicillin concentration on bacterial growth rate at $30^{\circ} \mathrm{C}$. Error bars in all three panels are standard error of the mean, though many are smaller than the data point shapes. 
(A)

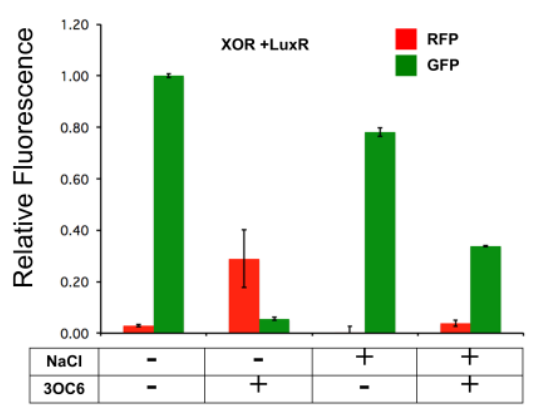

(B)

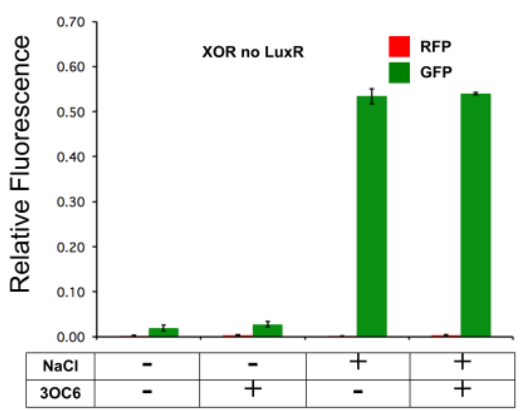

(C)

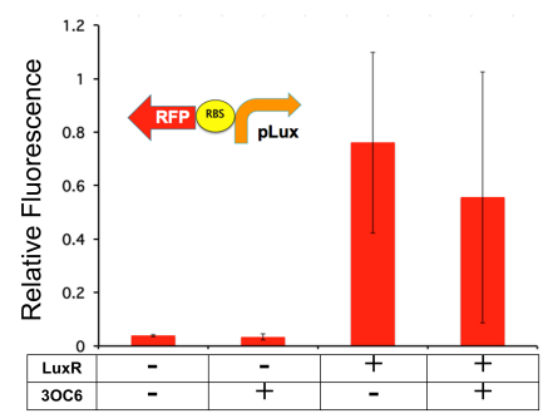

Figure 5. Testing XOR functionality. A) Relative GFP or RFP fluorescence for the XOR construct in the presence of the LuxR activator in all cells. Inputs of NaCl (high osmolarity) and $30 \mathrm{OC} 6$ as indicated. B) Relative GFP or RFP fluorescence for the XOR construct in the absence of LuxR in all cells. Inputs of NaCl (high osmolarity) and $30 \mathrm{OC} 6$ as indicated. C) Relative RFP fluorescence for a control construct designed to measure backwards transcription from $P_{\text {lux }}$ promoter. Addition of LuxR and $30 \mathrm{OC} 6$ as indicated. Error bars in all panels are one standard deviation.

coding system inside live cells compared to current hash functions based on complex hardware and algorithms. For certain applications, biological computation is superior to in silico computation because populations of cells can execute vast amounts of processes in parallel relatively inexpensively, and because of the cell's natural connection to the living world. For our bacterial hash function, we chose to implement an XOR logic gate because it integrates two inputs and provides equal distribution of outputs ${ }^{27}$. In addition to a potential hash function, biological logic gates have been studied for various other purposes and could be extremely useful in agriculture, production of pharmaceuticals and other products, and in medicine, particularly in the detection of cancer cells ${ }^{28}$.

In order to implement a simple hash function with a series of XOR logic gates, we designed and successfully demonstrated timedelayed growth of bacterial colonies based on the characteristic diffusion of B-lactamase in agar. Time-delayed bacterial growth ensures that each colony would be given sufficient time to unidirectionally process its inputs and perform XOR logic sequentially so that the message would be correctly hashed. An inexpensive and simple procedure for time-delayed growth could be a valuable tool for synthetic biologists who need signals to be passed to neighboring cells over a defined time period. As expected, we saw that bacterial growth rate decreased with a greater concentration of ampicillin. We incorrectly hypothesized that increased agar concentration would decrease bacterial growth rate because it would slow the diffusion of B-lactamase. After performing a literature search, we found that naficillin, which is structurally similar to ampicillin, is less effective when agar concentration is high $^{23}$. Sequestration of the antibiotic by agar would explain the increased bacterial growth rate we observed. The observation of a higher bacterial growth rate at higher temperature for the $25 \mu \mathrm{g} / \mathrm{mL}$ ampicillin concentration suggests that -lactamase enzyme function or diffusion is increased with temperature. Further studies should measure the effects of a wider range of temperatures and ampicillin concentration to understand the interaction between the two variables. Given that over production of -lactamase enhances its own secretion ${ }^{22}$, it would be informative to test the effect of plasmid copy-number on sequential bacterial growth, which could present an additional method of tuning the growth rate of bacteria. In one study, researchers found that employing low-copy plasmids and adding selective pressures with an antibiotic resistant gene significantly attenuated internal noise in gene circuits ${ }^{29}$. While we investigated the basic effects of initial ampicillin concentration, agar concentration, and temperature, further characterization is required to be able to fully develop and take advantage of time-delayed colony growth.

Our XOR logic gate was based on the opposition of the $\mathrm{P}_{\mathrm{OmpC}}$ and $\mathrm{P}_{\text {lux }}$ promoters. The designed logic gate did not function as predicted because of previously undocumented behavior of the BioBrick part containing the $P_{\text {lux }}$ promoter. In future studies, we would need to replace the $P_{\text {lux }}$ part with one that contains a unidirectional promoter that becomes activated only in the presence of its input signal. Candidate promoters include $P_{\text {tet }}, P_{\text {bad }}$, and $P_{\text {lacl, }}$ which have been studied for use in other biological logic gates ${ }^{11,30}$. Our results indicated that the $\mathrm{P}_{\text {lux }}$ promoter is bidirectional and is induced "backwards" by LuxR in the absence of 30c6. The backwards transcription in the absence of $30 \mathrm{OC} 6$ had not been documented in the literature ${ }^{14,15,24-26}$. In the $V$. fischeri genome, the $\mathrm{P}_{\text {lux }}$ promoter points in the direction of the Luxl gene and away from the LuxR gene. The native luxR gene has its own promoter "pointed to the left" but low levels of backwards transcription is possible from the adjacent $\mathrm{P}_{\text {lux }}$ promoter "pointed to the right" ${ }^{24}$. In these experiments, LuxR was produced by "backwards transcription" in the presence of LuxR and 30C6 but not with LuxR alone. The experiments that showed "backwards" promoter activity was 30c6dependent used the full $P_{\text {lux }}$ promoter that includes 70 additional bases compared to the $P_{\text {lux }}$ used in this study. In our experiments, the level of backwards transcription by $\mathrm{P}_{\text {lux }}$ in the presence of LuxR alone was greater than that of forwards transcription from $\mathrm{P}_{\text {lux }}$ in the presence of both LuxR and the inducer 30C6.

The current study suggests that in the absence of 30c6, transcription in the direction of LuxR might also produce a positive feedback loop for LuxR production ${ }^{31}$. Backwards transcription from the $P_{\text {lux }}$ promoter could be caused by attachment of LuxR to known binding sites in $\mathrm{P}_{\text {lux }}$. Alternatively, there could be a cryptic promoter in the $P_{\text {lux }}$ part that contains additional LuxR binding sites that have not yet been studied. The behavior of the LuxR regulatory protein may also be due to cytoplasmic differences between its native $\mathrm{Vi}$ brio fischeri bacterial cells and E. coli cells. The LuxR family of quorum-sensing proteins includes hundreds of orthologs across many species of prokaryotes. In nature, E. coli bacteria express a signal receptor in this family known as SdiA but do not possess an orthologous enzyme (such as Luxl) that produces signals (such as $30 \mathrm{OC}$ and other $\mathrm{N}$-acylhomoserine lactones[AHL]). Instead, E. coli is thought to detect and respond to the signals produced by other neighboring species, but this has been difficult to study ${ }^{32}$. A recent study by Dyszel, et al. (2010) demonstrated that sdiA is only partially dependent on AHL because plasmid-based sdiA induced a response in two important loci of $E$. coli, but not chromosome-based sdiA and/or $\mathrm{AHL}^{33}$. More research is necessary to investigate the mechanism of $\mathrm{AHL}$ detection in E. coli. Since the LuxR we transformed into $E$. coli was plasmid-based, the similarity to plasmidbased sdiA might influence the unpredicted behavior of $\mathrm{P}_{\text {lux. }}$ Perhaps $\mathrm{P}_{\text {lux }}$ should not be thought of as initiating "backwards" tran- 
scription, but $\mathrm{P}_{\text {lux }}$ with LuxR should be described as a toggle switch that is governed by the presence or absence of 30C6. In our experiments, the level of backwards transcription by $P_{l u x}$ in the presence of LuxR alone was greater than that of forwards transcription from $P_{\text {lux }}$ in the presence of both LuxR and the inducer $30 \mathrm{C6}$.

We have made progress towards a functional bacterial hash function with the development and testing of a novel design. We developed and characterized a method for inexpensive and simple timedelayed growth that is necessary for implementation of our hash function and is likely to find general utility in synthetic biology. Our DNA-based XOR logic gate did not function as expected, so we designed and built control constructs that provided data in support of more complete description of the molecular mechanism by which the LuxR activator controls bidirectional transcription from the commonly used $\mathrm{P}_{\text {lux }}$ promoter.

\section{Materials and Methods}

\section{Bacterial growth}

For measurement of time-delayed bacterial growth, LB agar plates were prepared by dissolving $1 \mathrm{~g}$ Bacto-tryptone, $5 \mathrm{~g}$ yeast extract, $10 \mathrm{~g} \mathrm{NaCl}$ in $800 \mathrm{~mL}$ water, adjusting the $\mathrm{pH}$ to 7.5 with $\mathrm{NaOH}$, adding agar, and adjusting the final volume to $1 \mathrm{~L}$. Agar added at a level of $7.5 \mathrm{~g}$ was designated $0.5 \mathrm{X}$, while $15 \mathrm{~g}$ was $1.0 \mathrm{X}$, and $22.5 \mathrm{~g}$ was $1.5 \mathrm{X}$. After sterilization, amplicillin was add to final concentrations of $25 \mu \mathrm{g} / \mathrm{mL}, 50 \mu \mathrm{g} / \mathrm{mL}$, or $100 \mu \mathrm{g} / \mathrm{mL}$. Each plate was inoculated in one corner with $2 \mu \mathrm{L}$ of an overnight culture of an amplicillin-resistant strain of $E$. coli. $2 \mu \mathrm{L}$ of amplicillin-sensitive JM109 E. coli overnight culture were inoculated. Plates were incubated at either $30^{\circ} \mathrm{C}$ or $37^{\circ} \mathrm{C}$ for still/video photodocumentation or measurement of the average distance to the farthest visible colony.

Testing of $\mathrm{P}_{\mathrm{OmpC}}$ used low and high osmolarity liquid media. LB media was prepared with $10 \mathrm{~g}$ of tryptone, $5 \mathrm{~g}$ of yeast extract, $5 \mathrm{~g}$ $\mathrm{NaCl}$ and $200 \mu \mathrm{l}$ of $5 \mathrm{M} \mathrm{NaOH}$ per liter of distilled water. TY liquid media was prepared with $10 \mathrm{~g}$ of tryptone and $5 \mathrm{~g}$ of yeast extract and no $\mathrm{NaCl}$ per liter of distilled water ${ }^{34}$. LB media was considered to be high osmolarity by comparison to the low osmolarity of the TY media, prepared without addition of $\mathrm{NaCl}$. Ampicillin was added to a final concentration of $100 \mu \mathrm{g} / \mathrm{ml}$. Testing of $P_{\text {lux }}$ required the autoinducer 30C6-HSL (Sigma-Aldrich Cat. \# K3007), added to a final concentration of $4 \mu \mathrm{g} / \mathrm{mL}$.

\section{Basic BioBrick assembly}

All DNA parts and sub-parts produced for this project were generated using the standard BioBrick assembly protocol using high copy plasmids pSB1AK3, pSB1A2 or pSB1A3 ${ }^{35}$, and registered on the MIT Registry of Standard Biological Parts ${ }^{36}$. The Promega Wizard Plus SV Minipreps (Cat. \#A1460) kit was used according to the manufacturer's instructions to extract plasmid DNA. BioBrick parts were digested with restriction enzymes, EcoRI, Xbal, Spel and Pstl to produce 'sticky ends' that were used to combine BioBrick parts. Digested DNA was purified using $1 \%$ agarose gel electrophoresis followed by gel purification with the QIAGEN QIAquick Gel Extraction Kit (Cat. \# 28706). BioBrick parts with matching 'sticky ends' were ligated using T4 ligase produced by Promega. Plasmid DNA was transformed into JM109 Z-Competent E. coli cells (Zymo Research Cat. \# T3003) according to the manufacturer's instructions. It is important to note that JM109 cells express the Lacl repressor, which inhibits pLac promoters ${ }^{37}$. Colonies from ligation reactions were screened by polymerase chain reaction (PCR). All PCR reactions in this project were performed using Promega GoTaq Green Master Mix (Cat. \# M7123) with the appropriate primers and template DNA. All final constructs were sequenced for verification.

\section{Construction of basic parts}

The 99 base pair $\mathrm{P}_{\mathrm{Ompc}}$ promoter (K199017) was cloned from the MC4100 strain of $E$. coli using polymerase chain reaction (PCR). Primers were synthesized by Eurofins MWG|Operon and designed using an online PCR primer design program ${ }^{38}$. The forward primer has the sequence 5' GCATGAATTCGCGGCCGCTTCTAGAGTTTA CATTTTGAAACATCTA 3'. The underlined portion is a $20 \mathrm{bp}$ sequence that is the first $20 \mathrm{bp}$ of the $\mathrm{P}_{\mathrm{Ompc}}$ promoter $^{19}$. The 5 , end of the primer is the standard BioBrick prefix ${ }^{35}$, consisting of three different restriction sites, EcoRI, Notl and Xbal, plus four bases (GCAT) to facilitate EcoRI digestion at the 5' end of the PCR product. The reverse primer has the sequence 5' GCATCTGCAGCGGC CGCTACTAGTAAGTCCATTCTCCCCAAAAATG 3'. The underlined portion is a $21 \mathrm{bp}$ sequence that is complementary to the last $21 \mathrm{bp}$ of the $\mathrm{P}_{\mathrm{Ompc}}$ promoter ${ }^{19}$. The $5^{\prime}$ end of the primer consists of the complement of the restriction sites of Spel, Notl and Pstl to form the standard BioBrick suffix ${ }^{35}$. The fours bases GCAT were added to the $5^{\prime}$ of the primer to facilitate Pstl digestion on the $3^{\prime}$ end of the PCR product (BBa_K199017). PCR was conducted using MC4100 strain of $E$. coli as a source of template. The PCR product was purified, ligated into a BioBrick vector, and transformed into JM109 cells. Putative clones were screened by DNA sequencing.

The 55 base pair $\mathrm{P}_{\text {lux }}$ promoter (BBa K199052), which includes the lux box ${ }^{39}$, was required to be opposite in orientation relative to $\mathrm{P}_{\text {Ompc }}$ in order to facilitate our XOR gate design (Figure 2). The $\mathrm{P}_{\text {lux }}$ reverse promoter $P_{\text {lux }- \text { rev }}$ was generated by assembling four smaller oligos together. The sequences for the four oligos were generated using the online "Oligo Cuts Optimization Program"40. The oligos were modified so that the two ends of $\mathrm{P}_{\text {lux }}$ were equivalent to BioBrick prefix and suffix that have been digested with EcoRI and Pstl. The oligos were produced by Eurofins MWG|Operon. The sequences of the oligos were 5' AATTCGCGGCCGCTTCTAGAGTTTATTCGACTA 3', 5' TAACAAACCATTTTCTTGCGTAAACC TGTACGATCCTACAGGTTACTAGTAGCGGCCGCTGCA 3', 5' TAC AGGTTTACGCAAGAAAATGGTTTGTTATAGTCGAATAAACTCTA GAAGCGGCCGCG 3', and 5' GCGGCCGCTACTAGTAACCTGTA GGATCG 3' to produce a 55 bp promoter with this sequence: 5' tttattcgactataacaaaccattttcttgcgtaaacctgtacgatcctacaggt 3 '. From left to right, the assembled promoter contains a -10 region, a spacer, a -35 region followed by one lux box which would classically initiate transcription to the left as written here ${ }^{39}$. The oligos were mixed with $1 \times$ annealing buffer [100 mM NaCl; $10 \mathrm{mM}$ Tris- $\mathrm{HCl}, \mathrm{pH} 7.4]$, so that the final concentration of each oligo was $5 \mu \mathrm{M}$. This solution was heated to $100^{\circ} \mathrm{C}$ and allowed to slowly cool to room temperature. The annealed oligos were ligated into a BioBrick vector, and transformed into JM109 cells. Putative clones were screened by DNA sequencing.

\section{Construction of composite parts}

The part RFP rev + RBS $_{\text {rev }}$ (BBa_K199021) was formed by ligating the pre-existing parts, RFP rev $(\mathrm{BBa} J 31008)$ and $\mathrm{RBS}_{\text {rev }}(\mathrm{BBa} \mathrm{J} 440$ 01). The parts $R_{F P}$ rev $+R B S_{\text {rev }}$ and RBS+GFP (BBa_E5500) were ligated to $P_{\text {lux }}\left(B B a \_R 0062\right)$ and $P_{\text {Ompc }}(K 199017)$ so that two constructs, $\mathrm{RFP}_{\text {rev }}+\mathrm{RBS}_{\text {rev }}$ (promoter) and (promoter)+RBS+GFP were built for each promoter, except for $\mathrm{P}_{\text {lux }}+\mathrm{RBS}+\mathrm{GFP}$ which had been previously built (PompC $-\mathrm{BBa}$ K199019; BBa_K199022; $\mathrm{P}_{\text {lux }}-\mathrm{BBa}$-K 199027). The purpose of the (promoter)+RBS+GFP constructs was to quantify the level of transcription in the direction the promoter is pointing, while the $\mathrm{RFP}_{\text {rev }}+\mathrm{RBS}_{\text {rev }}+$ (promoter) constructs was used to quantify the amount of transcription caused by each promoter in the reverse direction. Note that the pre-existing part (BBa K09100) containing $P_{\text {lux }}+$ RBS +GFP also contained $P_{\text {lac }}+R B S+L u x R+T T$ (TT is a transcription terminators) to the left of it because LuxR is needed for $\mathrm{P}_{\text {lux }}$ to be activated ${ }^{25}$.

For the experiment measuring forward activity, the constructs tested were $\mathrm{P}_{\mathrm{Ompc}}+\mathrm{RBS}+\mathrm{GFP}$ (BBa_K199019) and $\mathrm{P}_{\mathrm{lac}}+\mathrm{RBS}+$ LuxR+TT+ Plux +RBS+GFP+TT (BBa_K09100). BBa_K09100 was tested with and without IPTG and 30C6-HSL. For the experiment 
measuring backward activity, the constructs tested were $\mathrm{RFP}_{\text {rev }}+\mathrm{RBS}_{\text {rev }}+\mathrm{P}_{\text {Ompc }}(\mathrm{K} 199022), \mathrm{RFP}_{\text {rev }}+\mathrm{RBS}_{\text {rev }}+\mathrm{P}_{\text {lux }}$ (BBa_K19902 7 ), and $P_{\text {lac }}+$ RBS+RFP (BBa_J04450) as a control.

For construction of the XOR logic gate, the construct $\mathrm{RFP}_{\text {rev }}+\mathrm{RBS}_{\text {rev }}+\mathrm{P}_{\mathrm{OmpC}}(\mathrm{BBa} \mathrm{K} 199022)$ was ligated to $\mathrm{P}_{\text {lux-rev }}(\mathrm{BBa}$ $\mathrm{K} 199052)$ then to RBS+GFP (BBa E5500) to make the construct $\mathrm{RFP}_{\text {rev }}+\mathrm{RBS}_{\text {rev }}+\mathrm{P}_{\mathrm{OmpC}}+\mathrm{P}_{\text {lux-rev }}+\mathrm{RBS}+\mathrm{GFP}$ (BBa_K199069). LuxR expression with the XOR gate (BBa_K199104) was produced by ligating the XOR construct (BBa_K199069) to the right of $\mathrm{P}_{\text {lac }}$ + RBS+LuxR+TT (BBa K199103). LuxR production was induced when IPTG is added and activates $P_{\text {lac }}{ }^{41}$. However, it should be noted that $P_{\text {lac }}$ is known to be a leaky promoter with significant transcription activity even in the absence of IPTG ${ }^{37}$.

\section{Measuring fluorescence}

For measurement of fluorescence, $2 \mathrm{~mL}$ of liquid media was inoculated with the appropriate cells and incubated for 18 hours at $37^{\circ} \mathrm{C}$ with shaking. After incubation, $600 \mu \mathrm{L}$ of each construct-media combination was transferred to a microwell plate in $200 \mu \mathrm{L}$ triplicates. The microwell plate was analyzed using a fluorometer to obtain an absorbance reading and a fluorescence intensity for each $200 \mu \mathrm{L}$ sample. Each fluorescence intensity value was divided by its corresponding absorbance (595 $\mathrm{nm}$ ) reading to account for the varying levels of growth in each culture tube. Triplicates were then averaged. To measure green fluorescence, the fluorometer was set at $485 \mathrm{~nm}$ for excitation and $528 \mathrm{~nm}$ for emission measurement. To measure red fluorescence, the fluorometer was set at $540 \mathrm{~nm}$ for excitation and $600 \mathrm{~nm}$ for emission measurement.

\section{Acknowledgements}

We wish to thank the iGEM founders, organizers, and community for providing a supportive environment for conducting synthetic biology research with undergraduates and Paul Brantley for assistance with the figures. Support is gratefully acknowledged from NSF UBM grant DMS 0733952 to Davidson College and DMS 0733955 to Missouri Western State University, HHMI grants 52005120 and 52006292 to Davidson College, the James G. Martin Genomics Program at Davidson College, and the Missouri Western State University Foundation and Summer Research Institute. AMC, LJH, JLP and TTE are members of GCAT, the Genome Consortium for Active Teaching.

\section{References}

1. Schneier, B. (2008). America's next top hash function [http://www.wired.com/politics/security/commentary/securitymatt ers/2008/11/securitymatters_1120]. Wired.

2. Mackenzie, D. (2008). Computer science. Cryptologists cook up some hash for new 'bake-off'. Science 319, 1480-1481.

3. Barker, E., Bassham, L., Burr, W., Caswell, S., Chang, D., Chang, S.-j., Chen, L., Dang, Q., Dworkin, M., Kelsey, J., et al. (2009). Cryptographic hash algorithm competition. [http://www. nist.gov/itt/csd/ct/hash_competition.cfm]. The National Institute of Standards and Technology.

4. Tiwari, H., and Asawa, K. (2010). Cryptographic hash function: an elevated view. EJSR 43, 452-465.

5. Haynes, K.A., Broderick, M.L., Brown, A.D., Butner, T.L., Dickson, J.O., Harden, W.L., Heard, L.H., Jessen, E.L., Malloy, K.J., Ogden, B.J., et al. (2008). Engineering bacteria to solve the Burnt Pancake Problem. J Biol Eng 2, 8.

6. Baumgardner, J., Acker, K., Adefuye, O., Crowley, S.T., Deloache, W., Dickson, J.O., Heard, L., Martens, A.T., Morton, N., Ritter, M., et al. (2009). Solving a Hamiltonian Path Problem with a bacterial computer. J Biol Eng 3, 11.

7. Goni-Moreno, A., Redondo-Nieto, M., Arroyo, F., and Castella- nos, J. (2010). Biocircuit design through engineering bacterial logic gates. Nat Comput 10, 1007

8. de Silva, A.P., and McClenaghan, N.D. (2004). Molecular-scale logic gates. Chemistry 10, 574-586.

9. Western, D.-M. (2008). Our Models. [http://2008.igem.org/Team: Davidson-Missouri_Western/Our_Models] Accessed 25 May, 2011. iGEM.

10. Credi, A., Balzani, V., Langford, SJ., and Stoddart, JF. (1997). Logic operations at the molecular level: An XOR gate based on a molecular machine. J. Am. Chem. Soc. 119, 2679-2681.

11. Tamsir, A., Tabor, J.J., and Voigt, C.A. (2011). Robust multicellular computing using genetically encoded NOR gates and chemical 'wires'. Nature 469, 212-215.

12.Stojanovic, M.N., Mitchell, T.E., and Stefanovic, D. (2002). Deoxyribozyme-based logic gates. J Am Chem Soc 124, 35553561.

13. Kramer, B.P., Fischer, C., and Fussenegger, M. (2004). BioLogic gates enable logical transcription control in mammalian cells. Biotechnol Bioeng 87, 478-484.

14. Egland, K.A., and Greenberg, E.P. (2000). Conversion of the Vibrio fischeri transcriptional activator, LuxR, to a repressor. $J$ Bacteriol 182, 805-811.

15. Nasser, W., and Reverchon, S. (2007). New insights into the regulatory mechanisms of the LuxR family of quorum sensing regulators. Anal Bioanal Chem 387, 381-390.

16. Forst, S., Delgado, J., and Inouye, M. (1989). Phosphorylation of OmpR by the osmosensor EnvZ modulates expression of the ompF and ompC genes in Escherichia coli. Proc Natl Acad Sci U S A 86, 6052-6056.

17. Jung, K., Hamann, K., and Revermann, A. (2001). K+ stimulates specifically the autokinase activity of purified and reconstituted EnvZ of Escherichia coli. J Biol Chem 276, 40896-40902.

18. Cai, S.J., and Inouye, M. (2002). EnvZ-OmpR interaction and osmoregulation in Escherichia coli. J Biol Chem 277, 2415524161.

19. Yoshida, T., Qin, L., Egger, L.A., and Inouye, M. (2006). Transcription regulation of ompF and ompC by a single transcription factor, OmpR. J Biol Chem 281, 17114-17123.

20. OpenWetWare (2008). Ampicillin. (http://openwetware.org/wiki/ Ampicillin). Accessed 25 May, 2011.

21. Chandrakala, B., Elias, B.C., Mehra, U., Umapathy, N.S., Dwarakanath, P., Balganesh, T.S., and deSousa, S.M. (2001). Novel scintillation proximity assay for measuring membraneassociated steps of peptidoglycan biosynthesis in Escherichia coli. Antimicrob Agents Chemother 45, 768-775.

22. Georgiou, G., Shuler, M.L., and Wilson, D.B. (1988). Release of periplasmic enzymes and other physiological effects of betalactamase overproduction in Escherichia coli. Biotechnol Bioeng 32, 741-748

23. Toama, M.A., Issa, A.A., and Ashour, M.S. (1978). Effect of agar percentage, agar thickness, and medium constituents on antibiotics assay by disc diffusion method. Die Pharmazie 33, 100 102.

24. Shadel, G.S., and Baldwin, T.O. (1992). Positive autoregulation of the Vibrio fischeri luxR gene. LuxR and autoinducer activate cAMP-catabolite gene activator protein complex-independent and -dependent luxR transcription. J Biol Chem 267, 7696-7702.

25. Stevens, A.M., Dolan, K.M., and Greenberg, E.P. (1994). Synergistic binding of the Vibrio fischeri LuxR transcriptional activator domain and RNA polymerase to the lux promoter region. Proc Natl Acad Sci U S A 91, 12619-12623.

26. Long, T., Tu, K.C., Wang, Y., Mehta, P., Ong, N.P., Bassler, B.L., and Wingreen, N.S. (2009). Quantifying the integration of quorum-sensing signals with single-cell resolution. PLOS Biol 7, e68.

27. Manuel, S.a.S., N. (2007). XOR-Hash: A hash function based on XOR. WEWoRC 2007.

28. Anderson, J.C., Clarke, E.J., Arkin, A.P., and Voigt, C.A. (2006) Environmentally controlled invasion of cancer cells by engineered bacteria. J Mol Biol 355, 619-627. 
29. Sleight, S.C., Bartley, B.A., Lieviant, J.A., and Sauro, H.M. (2010). Designing and engineering evolutionary robust genetic circuits. J Biol Eng 4, 12.

30. Hasty, J., McMillen, D., and Collins, J.J. (2002). Engineered gene circuits. Nature 420, 224-230.

31. Dunlap, P.V. (1999). Quorum regulation of luminescence in Vibrio fischeri. J Mol Microbiol Biotechnol 1, 5-12.

32. Ahmer, B.M. (2004). Cell-to-cell signalling in Escherichia coli and Salmonella enterica. Mol Microbiol 52, 933-945.

33. Dyszel, J.L., Soares, J.A., Swearingen, M.C., Lindsay, A., Smith, J.N., and Ahmer, B.M. (2010). E. coli K-12 and EHEC genes regulated by SdiA. PLoS One 5, e8946.

34. Ohashi, K., Yamashino, T., and Mizuno, T. (2005). Molecular basis for promoter selectivity of the transcriptional activator OmpR of Escherichia coli: isolation of mutants that can activate the non-cognate kdpABC promoter. J Biochem 137, 51-59.

35. Knight, T. (2004). Idempotent Vector Design for Standard As sembly of Biobricks. MIT Articial Intelligence Laboratory, 1-11.

36. Retberg, R. (2005). MIT Registry of Standard Biological Parts $<$ http://partsregistry.org > . Accessed 8 May, 2011.

37. Kennell, D., and Riezman, H. (1977). Transcription and translation initiation frequencies of the Escherichia coli lac operon. $J$ Mol Biol 114, 1-21.

38. Barron, J., Parra, M, and Win, M. (2008). BioBrick PCR Primer Designer. (http://gcat.davidson.edu/iGEM08/bbprimer.html). Accessed 8 May, 2011. .

39. Urbanowski, M.L., Lostroh, C.P., and Greenberg, E.P. (2004). Reversible acyl-homoserine lactone binding to purified Vibrio fischeri LuxR protein. J Bacteriol 186, 631-637.

40. Harden, L. (2006). Oligo Cuts Optimization Program <http://gcat. davidson.edu/IGEM06/oligo.html>. Accessed 8 May, 2011. .

41. Jacob, F., Monod, J. (1961). On the regulation of gene activity. Cold Spring Harb Symp Quant Biol 26, 193-211. 